\title{
Genetic modification of ES cells to develop endoderm and islet progenitors
}

\author{
Pei Wang ${ }^{1}$, Ryan Rodriguez ${ }^{1}$, Xueying $\mathrm{Gu}^{1}$, Seung Kim ${ }^{1}$ \\ ${ }^{1}$ Department of Developmental Biology, Stanford University
}

In early vertebrate development, three germ layers are formed during gastrulation: the ectoderm, the mesoderm and the definitive endoderm. The definitive endoderm gives rise to the entire gastrointestinal epithelium as well as lung, liver, pancreas, biliary system, thyroid gland and thymus. Understanding how the endoderm forms from mouse and human ES cells is the first step towards the ultimate goal, generating specific cell types for therapeutic purposes, such as pancreatic b-cells. The molecular mechanisms of endoderm formation have been largely elucidated using model non-mammalian vertebrate systems. Less is known about endoderm formation in mammalians, including mice and humans. The expression of HMG box transcription factor Sox 17 has been reported to be characteristically found in definitive endoderm. Studies suggest that $S o x 17$ is required by embryonic progenitor cells to acquire endodermal fates and to differentiate properly into definitive endoderm. To study the differentiation of definitive endoderm in vitro and to rigorously assess differentiated cells, I have used homologous recombination to knockin EGFP into the Sox17 locus to generate mouse ES cells that will permit prospective isolation and enrichment of Sox17expressing cells. I have also generated mice lines using these genetically-modified ES cells, which will permit detailed investigation of the properties of Sox17-expressing definitive endoderm, including its molecular signature. Based on my success in achieving homologous recombination in human ES cells at the Oct4 locus, I have also made targeting constructs to insert an EGFP reporter into the Sox17 locus in human ES cells. I will examine Sox17-expressing endoderm-like cells derived from human ES cells. These studies will reveal the properties and potential of human ES cells directed toward endodermal fates, and should accelerate strategies to produce replacement islets for diabetes. Cell Research (2008) 18:s107. doi: 10.1038/cr.2008.197; published online 4 August 2008

Correspondence: Pei Wang

E-mail: peiwang@stanford.edu

Pei Wang, received her master degree from Shanghai Institute of Cell Biology and The Ph.D degree from Baylor College of Medicine. Now she is a Postdoc in Seung Kim lab at Stanford University. The focus of her research is in studying the properties of definitive endoderm and pancreatic precursors using genetic modified ES cells and transgenic mice, and developing novel strategies to generate insulin producing cells (IPCs) from embryonic stem (ES) cells. 